\title{
The Impact of Climate Change on Vaccine-Preventable Diseases: Insights From Current Research and New Directions
}

\author{
Ayesha S. Mahmud ${ }^{1}$ • Pamela P. Martinez ${ }^{2,3}$ - Jingxing He ${ }^{1} \cdot$ Rachel E. Baker $^{4}$ \\ Accepted: 6 October 2020 / Published online: 25 October 2020 \\ (C) Springer Nature Switzerland AG 2020
}

\begin{abstract}
Purpose of Review Vaccine-preventable diseases remain a major public health concern globally. Climate is a key driver of the dynamics of many infectious diseases, including those that are vaccine preventable. Understanding the impact of climate change on vaccine-preventable diseases is, thus, an important public health research priority. Here, we summarize the recent literature and highlight promising directions for future research.

Recent Findings Vaccine-preventable enteric diseases, such as cholera, exhibit sensitivity to precipitation and flooding events. The predicted increase in extreme weather events as a result of climate change could exacerbate outbreaks of these pathogens. For airborne pathogens, temperature and specific humidity have been shown to be the most important environmental drivers, although the impact of climate change on disease burden and dynamics remains unclear. Finally, the transmission dynamics of vector-borne diseases are dependent on both temperature and precipitation, and climate change is expected to alter the burden and geographic range of these diseases. However, understanding the interacting effects of multiple factors, including socioeconomic and ecological factors, on the vector-borne disease ecosystem will be a crucial step towards forecasting disease burden under climate change.

Summary Recent work has demonstrated associations between climate and transmission of vaccine-preventable diseases. Translating these findings into forecasts under various climate change scenarios will require mechanistic frameworks that account for both intrinsic and extrinsic drivers of transmission, and the non-linear effects on disease burden. Future research should also pay greater attention to uncertainty in both the climate modeling processes as well as disease outcomes in the context of vaccine-preventable diseases.
\end{abstract}

Keywords Environment $\cdot$ Climate change $\cdot$ Modeling $\cdot$ Vaccine-preventable diseases

\section{Introduction}

The role of weather and environmental factors in driving seasonal and multi-year outbreak cycles has been well-

This article is part of the Topical Collection on Climate Change and Health

Ayesha S. Mahmud mahmuda@berkeley.edu

1 University of California, Berkeley, Berkeley, CA, USA

2 Center for Communicable Disease Dynamics, Harvard T. H. Chan School of Public Health, Boston, USA

3 Department of Microbiology, University of Illinois at Urbana Champaign, Champaign, IL, USA

4 Princeton Environmental Institute, Princeton University, Princeton, NJ, USA documented for many pathogens. Environmental factors can shape the dynamics of infectious diseases through their effects on the abundance of vectors, the ability of the pathogen to survive outside the host, environmental contamination of water sources, and dampening of host immunity, as well as indirectly through disasters, such as droughts and floods, that can lead to population displacement, malnutrition and poor health, and disruptions in the health system $[1 \bullet, 2,3]$. Growing concerns about the impact of climate change have led to an increased interest in forecasting the future burden of infectious diseases under different climate change scenarios. Warming temperatures, changes in precipitation patterns, and in the severity and frequency of extreme weather events are likely to alter the range, spread, and burden of many infectious diseases, including vaccine-preventable diseases [4, 5] (Fig. 1).

Vaccines are one of the most important and effective public health tools available for combating the spread of infectious diseases. Yet, many vaccine-preventable diseases, such as 


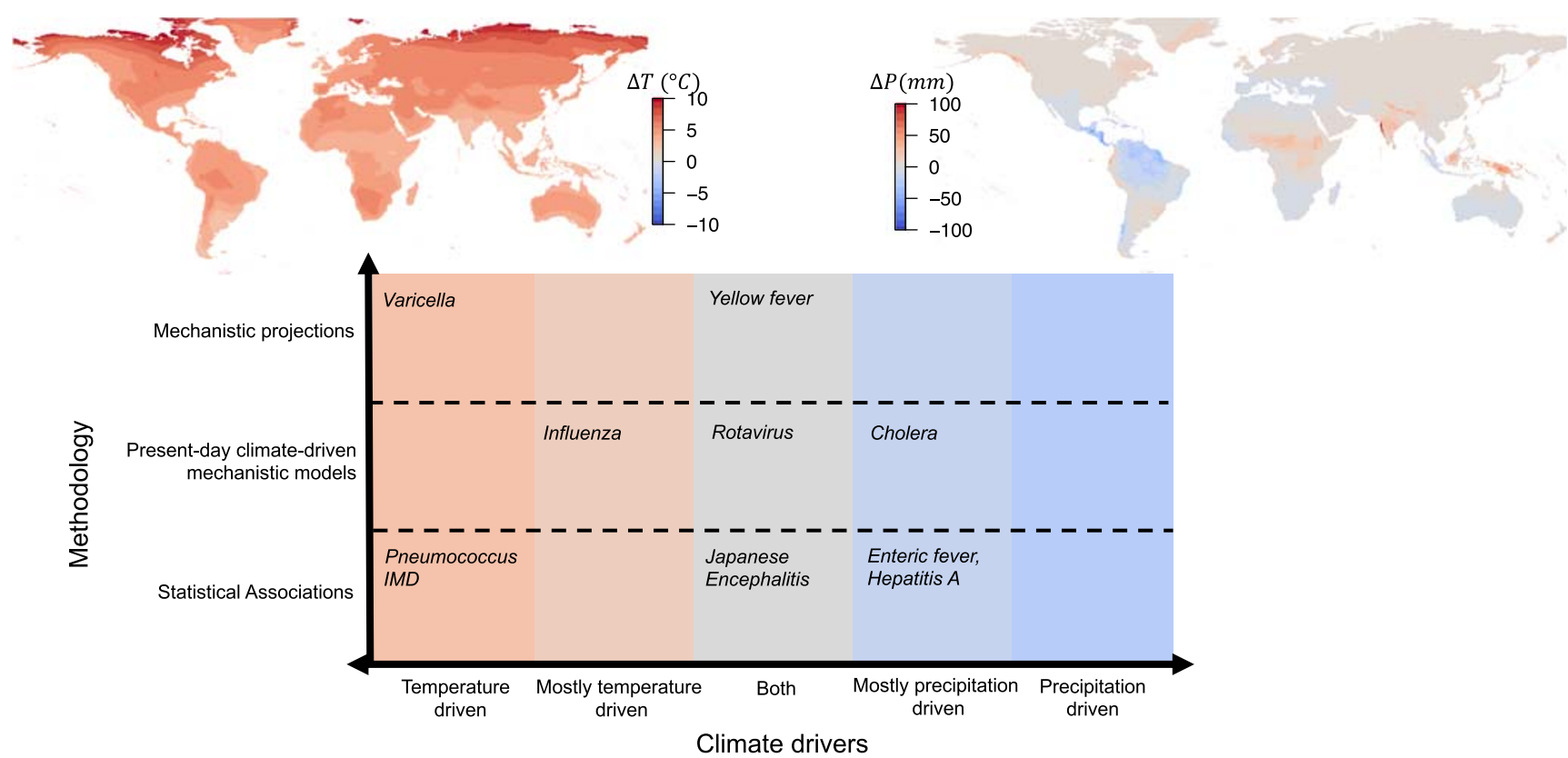

Fig. 1 Top panel: maps show the CMIP6 multi-model mean projected change in temperature $(\Delta T)$ and precipitation $(\Delta P)$ in 2100 relative to 2000 under the Shared Socioeconomic Pathway (SSP) 3 "middle of the road" scenario, generated using Worldclim data [6]. Bottom panel: plot shows a summary of climate drivers (temperature or precipitation) for different vaccine-preventable diseases and the "best case" modeling effort reviewed, where we assume the best case is a fully mechanistic model using projection data. Absolute humidity drivers are counted under temperature-driven given the functional dependence of the two variables measles and polio, have seen a global resurgence. Others, such as influenza and rotavirus, remain endemic in certain parts of the world, particularly in the tropics, with regular outbreaks seen globally. Understanding the role of climate change in the future dynamics of these diseases, and the implications for control and elimination strategies, is a key public health need.

The first step in predicting the impact of climate change on infectious diseases is to quantify the relationship between climate variables and disease burden or transmission. The research on the link between climate and infectious diseases typically falls into two broad categories (see Fig. 1 categorizing studies for vaccine-preventable diseases). The first uses a statistical framework to quantify the associations between climate variability and infectious disease burden using historical and current data. This body of work typically exploits spatial or temporal (seasonal or multi-annual) variation in climate variables to identify associations between these variations and heterogeneities in disease burden. The second uses a mechanistic understanding of the disease transmission process itself to make inferences about the impact of climate on disease burden. Because the dynamics of infectious diseases are inherently non-linear, driven by both extrinsic factors (such as climate) and intrinsic factors (such as population susceptibility), simple extrapolations to predict future burden using statistical associations between current climate and disease burden can be misleading. A mechanistic model-based framework that captures both intrinsic and extrinsic drivers of disease dynamics, as well as features of the host-pathogen biology, is necessary for forecasting the future burden of infectious diseases under different climate change scenarios.

Here, we summarize the recent literature on the impact of climate change on vaccine-preventable diseases, including both direct and indirect impacts, focusing on mechanistic frameworks where available. We highlight existing gaps in the literature and discuss directions for future research.

\section{Enteric Diseases}

The link between environmental factors and disease transmission is perhaps best understood for waterborne and enteric diseases that are sensitive to variations in precipitation. Climate change can lead to spatial and temporal changes in precipitation and rainfall patterns, and the occurrence of flooding, tropical storms, and droughts, all of which may impact water quality and the transmission of these pathogens.

The association between climatic factors and disease dynamics has long been known for cholera [7•, 8-13], which remains a major threat to public health in low-income countries, despite the existence of a vaccine. Cholera is caused by Vibrio cholerae, a bacterium that can survive and reproduce outside the host in aquatic environments. The interannual variations of temperature and precipitation are expected to be affected by climate change through changes in the intensity, frequency, and timing of climate anomalies [14]. El Niño Southern Oscillation (ENSO) is the main driver of climate 
variability and it has been shown to influence the population dynamics of cholera in places where it is endemic, such as Bangladesh [7•, 8-9], by increasing regional precipitation [10-12]. El Niño years, the warm phase of ENSO characterized by warm water in the Pacific Ocean, have been shown to modulate endemic cholera at highly localized urban settings $[13,15]$ and cause broad geographic shifts in cholera burden [16]. More generally, climate change can cause a rapid warming of coastal regions [17], leading to a potential expansion of the geographical extent of several pathogenic vibrio species, and consequently a higher disease burden.

Rotavirus, another vaccine-preventable enteric disease, also exhibits sensitivity to climatic conditions, but the role of climate change on its population dynamics remains unclear. Rotavirus is the most common cause of diarrhea among young children globally [18] and it exhibits significant heterogeneity in seasonality, suggesting the role of environmental drivers. Large seasonal outbreaks have been associated with low temperatures and low humidity, leading to a characteristic peak during the winter for most countries [19]. Regions that exhibit endemic transmission tend to have an additional peak during the summer for reasons that are not well understood [20,21]. Recent work using a mechanistic framework showed that monsoon flooding modulates the seasonality and interannual transmission of rotavirus in Bangladesh [22]. Thus, changes in precipitation and an increased frequency of flooding events as a result of climate change [4] may impact future burden in some parts of the world.

The role of climate variables in the transmission of other vaccine-preventable enteric diseases has primarily been addressed through statistical, rather than mechanistic, frameworks. Thus, while these statistical associations suggest that climate change is likely to have an impact on the burden and geographic range of these diseases, understanding the exact nature of this impact is a key direction for future research. Incidence of typhoid or enteric fever, caused by Salmonella enterica serovar Typhi and Paratyphi, has been positively associated with rainfall in Nepal [23], and humidity and temperature in Bangladesh [24]. However, a more recent analysis from Malawi shows a different picture, where an increased relative-risk of enteric fever was linked to both low and high temperatures, and only excessive rainfall $(\geq 13 \mathrm{~mm})$ was associated with a reduction in the disease [25]. For hepatitis A, another vaccinepreventable enteric disease, virus survival has been associated with lower temperatures [26], while extreme precipitation, El Niño years, and severe flood events have been linked to higher incidence [27-29]. In the case of polio, the effect of climatic factors on transmission is not well understood and, as a consequence, how future changes in climate will impact disease burden and eradication efforts remains an open question.

\section{Airborne Diseases}

The best evidence for the impact of climate on airborne pathogens comes from guinea pig animal models that have indicated a role for climate in modulating the transmission of influenza $[30,31]$. While initial results highlighted temperature and relative humidity as key drivers, specific humidity (the mass of water vapor in a unit mass of moist air) has been found to be the best fit predictor of transmission and is similarly correlated with virus survival [30]. Specific humidity has since successfully been used to predict influenza outbreaks in human populations $[32 \cdot, 33]$ with dry (low humidity) conditions leading to higher transmission. However, in tropical locations, precipitation may also play a role, though the mechanism linking precipitation to transmission is unclear [34, 35]. More recent work in Uganda provides evidence that influenza subtypes might have differential climate drivers [36]. Epidemics of influenza A/H1N1 were found to be associated with lower precipitation, but no association with climate was found for influenza/H3N2 and influenza B.

The potential distinct effects of precipitation and specific humidity have subtle implications when considering climate change. While specific humidity, driven by temperature changes, is expected to increase in the future, precipitation projections are more uncertain [37]. Although influenza has generally not been considered within a climate change framework, one study has suggested rapid weather variability (defined as the number of days in a 3-week period where day-today temperature changes were greater than $3 \mathrm{~K}$ ) could increase influenza mortality in the future [38] and another that higher temperatures could lead to a decline in the future [39]. Neither study, however, uses an epidemiological framework to derive future projections. Broadly, influenza findings have been supported by recent research on another airborne respiratory pathogen-respiratory syncytial virus (RSV) [40]. Although not yet vaccine-preventable, vaccines are in development for RSV, a disease with severe repercussions in young children. Climate drivers of RSV were found to be strikingly similar to influenza. Projections of climate change on RSV epidemic dynamics suggest that the intensity of outbreaks will decline in the future, but that severe outbreaks could occur during periods of high precipitation particularly in tropical locations and the western USA. Similarly, climate change projections have been generated for varicella, finding that higher relative humidity in the future is expected to lead to moderately more severe outbreaks [41]. In general, although there has been significant work relating climate drivers to infectious airborne diseases, further work is needed to generate projections under climate change scenarios, especially within a mechanistic framework.

While advances in influenza research have paved the way for understanding the climate drivers of airborne viruses, vaccine-preventable bacterial diseases have received less 
attention. There is some evidence of climate drivers for pneumococcus and invasive meningococcal disease (IMD) [42]. Seasonality has been detected in pneumococcal transmission, with wintertime conditions leading to higher transmission [43, 44]. For IMD, there is some evidence that incidence decreases as temperature increases or, similarly, that low humidity increases transmission $[42,45]$. The "meningitis belt" located in sub-Saharan Africa where IMD levels are high is of particular concern [46]. In this region, dry conditions, high winds, and increased dust may damage the upper respiratory tract, increasing transmission [42]. Accounting for these multiple potential drivers will complicate climate change projections for IMD: variables such as wind and dust levels are less frequently available in climate model output [47]. Nevertheless, the IPCC's fifth assessment report suggests the disease burden will be influenced by climate change [48].

\section{Vector-Borne Diseases}

There is a large body of literature that has examined the impact of weather and climate on vector-borne disease burden and transmission, particularly for mosquito-borne diseases. The dependence of mosquito populations on ecological (such as habitat suitability and land-use) and environmental factors (such as temperature and rainfall) mean that the burden of mosquito-borne diseases, and the prospects for their elimination, are strongly impacted by climate change. Climatic factors such as temperature and rainfall can affect mosquito abundance, survival, breeding, and biting rates [49, 50]. While temperature and precipitation are both important determinants of the vector life history, the interplay between these climatic factors are likely to have a more important effect than either variable alone [51]. Furthermore, the complexity of the vector-borne disease ecosystem (often involving non-human animal hosts), with many factors interacting and leading to non-linear effects means there is likely to be substantial uncertainty in projections of future disease burden as a result of changes in one or more of these factors. As highlighted in a recent review, there is currently no clear consensus on the impact of climate change on mosquito-borne diseases, possibly as a result of multiple interacting effects of other global change processes, such as land-use changes, which are often excluded from studies on climate and disease [49]. Understanding these complex, and often non-linear, interactions under various climate change scenarios is an important direction for future research.

The vast majority of the literature has focused on the impact of climate on malaria, which is transmitted by the Anopheles mosquito species, but recent work has also shown the importance of climatic factors for Aedes-borne diseases such as dengue. There has been less focus on the impact of climate on vaccine-preventable diseases that are transmitted via mosquitoes - yellow fever and Japanese Encephalitisalthough the incidence and global spread of these diseases are likely to be altered by climatic changes. Projections under climate change scenarios suggest a poleward shift in the distribution of Aedes mosquitoes, assuming that mosquito ranges will shift in response to changing temperatures and shifts in the geographic range of optimal temperatures. A nuanced examination by Ryan et al. [52॰] highlights differences in the patterns of projected geographic shifts for the two species of Aedes mosquitoes responsible for disease transmissionAedes albopictus and Aedes aegypti — due to differing thermal niche for the two vectors. They find that climate change will lead to increases in the total number of people exposed to Aedes-borne viruses, and lead to the expansion of the virus to new geographic regions. For the Albopictus species, however, they find that the most extreme increase in transmission globally is predicted to occur at intermediate climate change scenarios, highlighting the need for detailed projections by vector species.

Although the geographic range of Japanese encephalitis and yellow fever is also likely to change with climate, and possibly in a similar way as that predicted for dengue, there is a need for future research to focus more specifically on these diseases. The Japanese encephalitis virus (JEV) is a flavivirus belonging to the same genus as dengue but is transmitted to humans through bites from infected mosquitoes of the Culex species (mainly Culex tritaeniorhynchus). The transmission cycle of JEV includes mosquitoes, pigs, and/or water birds, and the disease is predominantly found in settings where humans live in close proximity to these animal hosts. The expansion of vaccination programs, improvements in living standards, and the reduced contact between humans and animals due to mechanization of agriculture has led to reduction of JEV in many countries, although the disease is endemic in 24 countries in parts of Asia and the Pacific [53]. In countries with endemic transmission, outbreaks typically occur during the warm season (in temperate regions) and during the rainy season (in tropical and subtropical regions), as well as during the pre-harvest period in rice-cultivating regions [53]. The link between climate and JEV, however, has not been well-studied and the impact of climate change remains an open question. Because the enzootic cycle of JEV, and the interaction between humans and animal hosts, is a key driver of JEV epidemics, other large-scale global changes, such as demographic shifts and land-use changes, are likely to have a large impact on JEV transmission in the future.

Yellow fever is an acute viral hemorrhagic disease caused by another flavivirus - the yellow fever virus (YFV) - and is transmitted by mosquitoes, belonging to the Aedes and Haemagogus species. YFV is endemic primarily in tropical regions of Africa and Latin America, with about $90 \%$ of cases occurring in Africa. The disease has three transmission cycles, all of which may be impacted by climate change: (1) a sylvatic 
cycle where transmission is maintained in non-human primate populations by mosquitoes, (2) an intermediate cycle where transmission occurs between humans and non-human primates via peri-domestic mosquitoes, and (3) an urban and peri-urban cycle where human-to-human transmission is propagated by Aedes aegypti mosquitoes [51].

Climatic variables such as temperature and rainfall have been shown to be significantly correlated with the global distribution of YFV [51,54], and previous studies have highlighted the role of seasonality in climatic factors in driving outbreaks [55]. Specifically, the interaction between temperature suitability and rainfall has been shown to account for much of the variability of YFV transmission across Africa [51]. The existence of a sylvatic cycle for YFV complicates the picture as climate change is likely to alter the interactions of mosquito species with non-human primates, and could lead to the evolution of new serotypes thereby compromising vaccination programs [56]. The risk of yellow fever spillover from non-human primates has been shown to be associated with rainfall-driven seasonality in the vector populations and temperature-driven seasonality in vector survival and infectiousness [55]. The complex interactions between global changes such as climate change, demographic change, and land-use change are likely to have an impact on YFV evolution and the burden of yellow fever globally. Understanding the role of environmental factors on both the sylvatic and domestic cycles of YFV, as well as the role of environmental factors in shaping virus phenotypic variance, will be a key element of understanding the overall impact of climate change on YFV [56].

\section{Indirect Pathways Linking Climate and Infectious Diseases}

Beyond the direct impact on transmission, climate change is also expected to have indirect impacts on the burden of vaccine-preventable diseases primarily through the expected increase in the frequency, geographic range, and severity of extreme weather events. More frequent extreme weather events, as a result of climate change, can exacerbate the spread of infectious diseases by displacing populations, damaging the public health infrastructure, disrupting access to routine public health services, including vaccination, and destroying local sanitation infrastructure and water systems [3, 57, 58]. Displaced populations, with reduced access to healthcare and often living in crowded conditions, are at a higher risk of experiencing infectious disease outbreaks [59]. Measles and meningitis outbreaks occurred after the 2004 tsunami and flooding in Banda Aceh [60]. Cholera outbreaks have been reported in Mozambique following hurricanes in 2019 [57, 61], in Haiti following Hurricane Mathew [62], and in West Bengal following the 1998 floods [63]. The disruption of routine medical care such as vaccinations for children can also lead to subsequent outbreaks of common vaccinepreventable diseases. These disruptions could impact the transmission of pathogens that are not considered to be directly impacted by climate change, such as human papillomavirus, measles, mumps, and rubella. Finally, while current research has not explicitly addressed the potential impact of climate change on vaccine delivery systems, the increasing frequency of extreme heat and other weather events could cause disruptions to vaccine thermostability and the cold chain system, particularly in remote areas [64].

\section{Conclusions and Future Research Directions}

Vaccine-preventable diseases remain a major cause of mortality and morbidity globally. Elimination and eradication efforts for vaccine-preventable diseases have been hampered by a multitude of factors including difficulties in developing vaccines with high efficacy for some diseases (such as influenza), vaccine refusal, and vaccine distribution issues. In light of these barriers to elimination, there is a growing interest in understanding the impact of climate change on the transmission of vaccine-preventable diseases. Climatic factors have been shown to be important drivers of vaccine-preventable respiratory airborne diseases, water-borne enteric diseases, and mosquito-borne diseases, yet much work remains in translating these findings to forecast disease burden under climate change. We have identified three major directions of future research to address existing gaps in the literature. First, we advocate for a greater focus on mechanistic models of disease transmission. Accurately attributing the impact of climate drivers on disease transmission is challenging due to the existence of multiple drivers of transmission, many of which interact in complex ways and produce synergistic effects on disease burden. Developing mechanistic frameworks that account for intrinsic and extrinsic drivers, and these non-linear effects, is thus a crucial step in generating reliable forecasts under different climate change scenarios.

Second, accounting for the interacting effects of other global change processes, such as changes in land-use, socioeconomic conditions, and demography, is important for developing a holistic understanding of the impact of climate change on disease burden. This will be particularly important for understanding the impact of climate change on zoonoses and the emergence of novel pathogens due to spillover events. The emergence of SARS-CoV-2 and the resulting global pandemic have highlighted the importance of studying these spillover events. Additionally, socioeconomic conditions, as well as disruptions of the public health infrastructure and vaccine distribution, either as a result of climate change or otherwise, will have an impact on disease burden. The majority of current research, however, has studied the impact of climate change in 
isolation, and examining the impact of climate change in conjunction with changes in various ecological, environmental, and socioeconomic factors should be a key direction for future research.

Third, projections of future epidemics need to account for uncertainty in both the climate modeling processes as well as disease outcomes. Sources of uncertainty include differences in projections from ensemble member climate models, differences across climate change scenarios, and uncertainty in climate-transmission estimates [65]. The vast majority of current studies focus on the output of a single climate model or uses only the multi-model mean. While this may be sufficient for temperature projections, where there is broad agreement, precipitation projections may vary significantly across models.

The output of the latest IPCC-driven climate modeling effort, Coupled Model Intercomparison Project (CMIP) 6, is newly available (Fig. 1). Projections in this case are based on the recently developed Shared Socioeconomic Pathways [66]. The SSPs present scenarios of possible economic, demographic, and environmental change, with the advantage that accompanying population projections have also been developed [67]. This presents a novel opportunity for incorporating both population changes and climate changes into future projections of infectious disease. With resources, such as Worldclim [6], providing access to downscaled and biascorrected versions of the climate projection data, across the range of climate model output, accounting for uncertainty is increasingly feasible. More broadly, these novel data streams present a unique opportunity to address key questions of climate change and infectious disease going forward.

\section{Compliance with Ethical Standards}

Conflict of Interest The authors declare that they have no conflicts of interest.

Human and Animal Rights and Informed Consent This article does not contain any studies with human or animal subjects performed by any of the authors.

\section{References}

Papers of particular interest, published recently, have been highlighted as:

- Of importance

1. Metcalf CJE, Walter KS, Wesolowski A, Buckee CO, Shevliakova E, Tatem AJ, et al. Identifying climate drivers of infectious disease dynamics: recent advances and challenges ahead. Proc Biol Sci. 2017;284. https://doi.org/10.1098/rspb.2017.0901 This review paper describes the mechanisms through which climate affects infectious diseases, and the challenges and recent advances in modeling these relationships.
2. Patz JA, Epstein PR, Burke TA, Balbus JM. Global climate change and emerging infectious diseases. JAMA. 1996;275:217-23.

3. Colwell RR, Patz JA. Climate, Infectious disease and health: an interdisciplinary perspective. 1998 [cited 6 May 2020]. Available: h t t p s:// w w w. s e mantics cholar.org/paper/ a00567037684beba2b64e2505e2e6ab907205424

4. Stocker TF, Qin D, Plattner G-K, Tignor M, Allen SK, Boschung J, et al. Climate change 2013: the physical science basis. Contribution of working group I to the fifth assessment report of the intergovernmental panel on climate change 2013;1535. Available: http:/ www.climatechange2013.org/images/report/WG1AR5 Frontmatter_FINAL.pdf

5. Seneviratne SI, Nicholls N, Easterling D, Goodess CM, Kanae S, Kossin J, et al. Changes in climate extremes and their impacts on the natural physical environment. 2017. Available: https://stgwedocs.unep.org/bitstream/handle/20.500.11822/18482/Climate Change_2014_Impacts_Adaptation_and_V.pdf?sequence $=1$

6. Fick SE, Hijmans RJ. WorldClim 2 : new 1-km spatial resolution climate surfaces for global land areas. Int J Climatol. 2017;37: 4302-15.

7. Pascual M, Rodó X, Ellner SP, Colwell R, Bouma MJ. Cholera dynamics and El Niño-Southern Oscillation. Science. 2000;289: 1766-9 This study was the first linking the role of environmental variables with the interannual variability of cholera dynamics.

8. Koelle K, Rodó X, Pascual M, Yunus M, Mostafa G. Refractory periods and climate forcing in cholera dynamics. Nature. 2005;436: 696-700.

9. Rodo X, Pascual M, Fuchs G, Faruque ASG. ENSO and cholera: a nonstationary link related to climate change? Proc Natl Acad Sci U S A. 2002;99:12901-6.

10. Cash BA, Rodó X, Kinter JL. Links between tropical Pacific SST and cholera incidence in Bangladesh: role of the eastern and central tropical Pacific. J Clim. 2008:4647-63. https://doi.org/10.1175/ 2007 jcli2001.1.

11. Cash BA, Rodó X, Kinter JL. Links between tropical Pacific SST and cholera incidence in Bangladesh: role of the western tropical and central extratropical Pacific. J Clim. 2009. pp. 1641-1660. https://doi.org/10.1175/2008jcli2177.1

12. Cash BA, Rodó X, Kinter JL, Yunus M. Disentangling the impact of ENSO and Indian Ocean variability on the regional climate of Bangladesh: implications for cholera risk. J Clim. 2010:2817-31. https://doi.org/10.1175/2009jcli2512.1.

13. Reiner RC, King AA, Emch M, Yunus M, Faruque ASG, Pascual M. Highly localized sensitivity to climate forcing drives endemic cholera in a megacity. Proceedings of the National Academy of Sciences. 2012. pp. 2033-2036. https://doi.org/10.1073/pnas. 1108438109

14. Intergovernmental Panel on Climate Change. Managing the risks of extreme events and disasters to advance climate change adaptation: special report of the intergovernmental panel on climate change. Cambridge University Press; 2012.

15. Martinez PP, Reiner RC Jr, Cash BA, Rodó X, Shahjahan Mondal M, Roy M, et al. Cholera forecast for Dhaka, Bangladesh, with the 2015-2016 E1 Niño: lessons learned. PLoS One. 2017;12: $\mathrm{e} 0172355$.

16. Moore SM, Azman AS, Zaitchik BF, Mintz ED, Brunkard J, Legros D, et al. El Niño and the shifting geography of cholera in Africa. Proc Natl Acad Sci U S A. 2017;114:4436-41.

17. Lima FP, Wethey DS. Three decades of high-resolution coastal sea surface temperatures reveal more than warming. Nat Commun. 2012;3:704.

18. Tate JE, Burton AH, Boschi-Pinto C, Parashar UD. Global, regional, and national estimates of rotavirus mortality in children $<5$ years of age, 2000-2013. Clin Infect Dis. 2016:S96-S105. https://doi. org/10.1093/cid/civ1013. 
19. D'Souza RM, Hall G, Becker NG. Climatic factors associated with hospitalizations for rotavirus diarrhoea in children under 5 years of age. Epidemiol Infect. 2008;136:56-64.

20. Levy K, Hubbard AE, Eisenberg JNS. Seasonality of rotavirus disease in the tropics: a systematic review and meta-analysis. Int $\mathrm{J}$ Epidemiol. 2009;38:1487-96.

21. Patel MM, Pitzer VE, Alonso WJ, Vera D, Lopman B, Tate J, et al. Global seasonality of rotavirus disease. Pediatr Infect Dis J. 2013;32:e134-47.

22. Martinez PP, King AA, Yunus M, Faruque ASG, Pascual M. Differential and enhanced response to climate forcing in diarrheal disease due to rotavirus across a megacity of the developing world. Proceedings of the National Academy of Sciences. 2016. pp. 4092 4097. doi:https://doi.org/10.1073/pnas.1518977113

23. Karkey A, Arjyal A, Anders KL, Boni MF, Dongol S, Koirala S, et al. The burden and characteristics of enteric fever at a healthcare facility in a densely populated area of Kathmandu. PLoS ONE. 2010. p. e13988. doi:https://doi.org/10.1371/journal.pone.0013988

24. Chowdhury FR, Ibrahim QSU, Bari MS, Alam MMJ, Dunachie SJ, Rodriguez-Morales AJ, et al. The association between temperature, rainfall and humidity with common climate-sensitive infectious diseases in Bangladesh. PLoS One. 2018;13:e0199579.

25. Thindwa D, Chipeta MG, Henrion MYR, Gordon MA. Distinct climate influences on the risk of typhoid compared to invasive non-typhoid Salmonella disease in Blantyre. Malawi Sci Rep. 2019;9:20310

26. Rose JB, Epstein PR, Lipp EK, Sherman BH, Bernard SM, Patz JA. Climate variability and change in the United States: potential impacts on water- and foodborne diseases caused by microbiologic agents. Environ Health Perspect. 2001:211. https://doi.org/10.2307/ 3435011.

27. $\mathrm{Hu}$ W, McMichael AJ, Tong S. El Niño Southern Oscillation and the transmission of hepatitis A virus in Australia. Med J Aust. 2004; 180:487-8.

28. Gao L, Zhang Y, Ding G, Liu Q, Wang C, Jiang B. Projections of hepatitis A virus infection associated with flood events by 2020 and 2030 in Anhui Province, China. Int J Biometeorol. 2016;60:187384.

29. Gullón P, Varela C, Martínez EV, Gómez-Barroso D. Association between meteorological factors and hepatitis A in Spain 2010 2014. Environ Int. 2017:230-5. https://doi.org/10.1016/j.envint. 2017.03.008

30. Shaman J, Kohn M. Absolute humidity modulates influenza survival, transmission, and seasonality. Proc Natl Acad Sci U S A. 2009;106:3243-8.

31. Lowen AC, Steel J. Roles of humidity and temperature in shaping influenza seasonality. J Virol. 2014;88:7692-5.

32. Shaman J, Pitzer VE, Viboud C, Grenfell BT, Lipsitch M. Absolute humidity and the seasonal onset of influenza in the continental United States. PLoS Biol. 2010;8:e1000316 This study on absolute humidity and influenza transmission bridges scales between laboratory estimates and population-level predictions using a mechanistic framework.

33. Yang W, Lipsitch M, Shaman J. Inference of seasonal and pandemic influenza transmission dynamics. Proc Natl Acad Sci U S A. 2015;112:2723-8.

34. Tamerius JD, Shaman J, Alonso WJ, Bloom-Feshbach K, Uejio $\mathrm{CK}$, Comrie A, et al. Environmental predictors of seasonal influenza epidemics across temperate and tropical climates. PLoS Pathog. 2013;9:e1003194.

35. Tamerius J, Nelson MI, Zhou SZ, Viboud C, Miller MA, Alonso WJ. Global influenza seasonality: reconciling patterns across temperate and tropical regions. Environ Health Perspect. 2011;119: 439-45.

36. Yang W, Cummings MJ. Dynamics of influenza in tropical Africa: temperature, humidity, and co-circulating (sub) types. Influenza
Other Respir Viruses. 2018. Available: https://onlinelibrary.wiley. com/doi/abs/10.1111/irv.12556;12:446-56.

37. Collins M, Knutti R, Arblaster J, Dufresne J-L, Fichefet T, Friedlingstein $\mathrm{P}$, et al. Long-term climate change: projections, commitments and irreversibility. Climate Change 2013-The Physical Science Basis: Contribution of Working Group I to the Fifth Assessment Report of the Intergovernmental Panel on Climate Change. Cambridge University Press; 2013. pp. 1029-1136.

38. Liu Q, Tan Z-M, Sun J, Hou Y, Fu C, Wu Z. Changing rapid weather variability increases influenza epidemic risk in a warming climate. Environ Res Lett. 2020;15:044004.

39. Ballester J, Rodó X, Robine J-M, Herrmann FR. European seasonal mortality and influenza incidence due to winter temperature variability. Nat Clim Chang. 2016;6:927-30.

40. Baker RE, Mahmud AS, Wagner CE, Yang W, Pitzer VE, Viboud $\mathrm{C}$, et al. Epidemic dynamics of respiratory syncytial virus in current and future climates. Nat Commun. 2019;10:5512.

41. Baker RE, Mahmud AS, Metcalf CJE. Dynamic response of airborne infections to climate change: predictions for varicella. Clim Chang. 2018;148:547-60.

42. Palmgren H. Meningococcal disease and climate. Glob Health Action. 2009;2:2061. https://doi.org/10.3402/gha.v2i0.2061.

43. Numminen E, Chewapreecha C, Turner C, Goldblatt D, Nosten F, Bentley SD, et al. Corrigendum: climate induces seasonality in pneumococcal transmission. Sci Rep. 2016;6:23307.

44. de Cellès MD, Arduin H, Lévy-Bruhl D, Georges S, Souty C, Guillemot D, et al. Unraveling the seasonal epidemiology of pneumococcus. Proc Natl Acad Sci U S A. 2019;116:1802-7.

45. Vescio F, Busani L, Mughini Gras L, Fazio C, Neri A, Avellis L, et al. Climate, demographic factors and geographical variations in the incidence of invasive meningococcal disease in Italy. Epidemiol Infect. 2015;143:1742-50.

46. Molesworth AM, Cuevas LE, Connor SJ, Morse AP, Thomson MC. Environmental risk and meningitis epidemics in Africa. Emerg Infect Dis. 2003;9:1287-93.

47. Fick SE, Hijmans RJ. WorldClim 2: new 1-km spatial resolution climate surfaces for global land areas. Int J Climatol. 2017. Available: https://rmets.onlinelibrary.wiley.com/doi/abs/10.1002/ joc. 5086 ? cas a token=BSV-ga $7 \mathrm{wjN} 8$ A A A A A : uo 4 hufUCzTeB 3 bXKNPm 1 LDHOabne 0 B C Tr 5 R oWdu0LhZEq7oNAwXm5_JzI9XswXj9TFS9HbeFO8rdGg;37: 4302-15.

48. Pachauri RK, Allen MR, Barros VR, Broome J, Cramer W, Christ R, et al. Climate change 2014: synthesis report. Contribution of Working Groups I, II and III to the Fifth Assessment Report of the Intergovernmental Panel on Climate Change. Pachauri RK, Meyer L, editors. Geneva, Switzerland: IPCC; 2014. p. 151.

49. Franklinos LHV, Jones KE, Redding DW, Abubakar I. The effect of global change on mosquito-borne disease. Lancet Infect Dis. 2019;19:e302-12.

50. Mordecai EA, Caldwell JM, Grossman MK, Lippi CA, Johnson LR, Neira M, et al. Thermal biology of mosquito-borne disease. Ecol Lett. 2019;22:1690-708.

51. Hamlet A, Jean K, Perea W, Yactayo S, Biey J, Van Kerkhove M, et al. The seasonal influence of climate and environment on yellow fever transmission across Africa. PLoS Negl Trop Dis. 2018;12: e0006284.

52. Ryan SJ, Carlson CJ, Mordecai EA, Johnson LR. Global expansion and redistribution of Aedes-borne virus transmission risk with climate change. PLoS Negl Trop Dis. 2019;13:e0007213 This study uses an empirically parametrized transmission model to forecast the burden of Aedes-borne viral diseases under different climate change scenarios.

53. Erlanger TE, Weiss S, Keiser J, Utzinger J, Wiedenmayer K. Past, present, and future of Japanese encephalitis. Emerg Infect Dis. 2009;15:1-7. 
54. Althouse BM, Hanley KA, Diallo M, Sall AA, Ba Y, Faye O, et al. Impact of climate and mosquito vector abundance on sylvatic arbovirus circulation dynamics in Senegal. Am J Trop Med Hyg. 2015;92:88-97.

55. Childs ML, Nova N, Colvin J, Mordecai EA. Mosquito and primate ecology predict human risk of yellow fever virus spillover in Brazil. Philos Trans R Soc Lond Ser B Biol Sci. 2019;374:20180335.

56. Tabachnick WJ. Climate change and the Arboviruses: lessons from the evolution of the dengue and yellow fever viruses. Annu Rev Virol. 2016;3:125-45.

57. Kahn R, Mahmud AS, Schroeder A, Aguilar Ramirez LH, Crowley $\mathrm{J}$, Chan J, et al. Rapid forecasting of cholera risk in Mozambique: translational challenges and opportunities. Prehosp Disaster Med. 2019;34:557-62.

58. McMichael AJ. Extreme weather events and infectious disease outbreaks. Virulence. 2015;6:543-7.

59. Hammer CC, Brainard J, Hunter PR. Risk factors and risk factor cascades for communicable disease outbreaks in complex humanitarian emergencies: a qualitative systematic review. BMJ Glob Health. 2018;3:e00647.

60. Kouadio IK, Aljunid S, Kamigaki T, Hammad K, Oshitani H. Infectious diseases following natural disasters: prevention and control measures. Expert Rev Anti-Infect Ther. 2012;10:95-104.

61. Cambaza E, Mongo E, Anapakala E, Nhambire R, Singo J, Machava E. Outbreak of cholera due to Cyclone Kenneth in northern Mozambique, 2019. Int J Environ Res Public Health. 2019;16. https://doi.org/10.3390/ijerph16162925.
62. Hulland E, Subaiya S, Pierre K, Barthelemy N, Pierre JS, Dismer A, et al. Increase in reported cholera cases in Haiti following Hurricane Matthew: an interrupted time series model. Am J Trop Med Hyg. 2019:368-73. https://doi.org/10.4269/ajtmh.17-0964.

63. Jutla A, Khan R, Colwell R. Natural disasters and cholera outbreaks: current understanding and future outlook. Curr Environ Health Rep. 2017;4:99-107.

64. Guo B, Naish S, Hu W, Tong S. The potential impact of climate change and ultraviolet radiation on vaccine-preventable infectious diseases and immunization service delivery system. Expert Rev Vaccines. 2015;14:561-77.

65. Visser H, Folkert RJM, Hoekstra J, De Wolff JJ. Identifying key sources of uncertainty in climate change projections. Clim Chang. 2000;45:421-57.

66. O'Neill BC, Kriegler E, Riahi K, Ebi KL, Hallegatte S, Carter TR, et al. A new scenario framework for climate change research: the concept of shared socioeconomic pathways. Clim Chang. 2014;122:387-400.

67. Jones B, O’Neill BC. Spatially explicit global population scenarios consistent with the shared socioeconomic pathways. Environ Res Lett. 2016;11:084003.

Publisher's Note Springer Nature remains neutral with regard to jurisdictional claims in published maps and institutional affiliations. 